\title{
On the existence of impulsive neutral evolution differential inclusions with infinite delay
}

\author{
Kai-Biao Lin ${ }^{1}$, Hsiang Liu ${ }^{2}$ and Chin-Tzong Pang ${ }^{3 *}$
}

"Correspondence:
imctpang@saturn.yzu.edu.tw
${ }^{3}$ Department of Information
Management, Innovation Center for
Big Data and Digital Convergence,
Yuan Ze University, Chung-Li, 32003,
Taiwan
Full list of author information is
available at the end of the article

available at the end of the article

\begin{abstract}
In this paper, we use a fixed point principle regarding a condensing mapping with some measure of noncompactness. We assume there is no compactness assumption, and we give sufficient conditions for the existence of mild solutions to some classes of impulsive neutral evolution differential inclusions with infinite delay. A concrete example is presented in the end to illustrate the abstract theorem.
\end{abstract}

MSC: 34K30; 34K40; 34G25; 47H04; 47H10

Keywords: condensing mapping; evolution family; fixed point principle; impulsive neutral evolution differential inclusions; infinite delay; measure of noncompactness

\section{Introduction}

In this paper, we are concerned with the existence of a mild solution of impulsive neutral evolution differential inclusions with infinite delay in a Banach space $X$. More precisely, we consider the following class of evolution systems:

$$
\left\{\begin{array}{l}
\frac{d}{d t}\left[x(t)+F\left(t, x_{t}\right)\right] \in A(t) x(t)+G\left(t, x_{t}\right), \quad t \in[0, T], t \neq t_{i} \\
\Delta x\left(t_{i}\right)=I_{i}\left(x_{t_{i}}\right), \quad i=1,2, \ldots, m, \\
x_{0}=\varphi \in \mathcal{B}
\end{array}\right.
$$

where $T>0, A(t): D \subset X \rightarrow X$ are linear closed operator in a Banach space $(X,\|\cdot\|), 0<$ $t_{1}<\cdots<t_{i}<\cdots<t_{m}<T$ are pre-fixed numbers; the history $x_{t}:(-\infty, 0] \rightarrow X, x_{t}(s)=x(t+$ $s)$, belongs to some abstract phase space $\mathcal{B}$ which will be defined with axioms introduced by Hale and Kato [1]; $F:[0, T] \times \mathcal{B} \rightarrow X$ is some suitable function; $G$ is a multimap from $[0, T] \times \mathcal{B}$ to the collection of all nonempty, compact, and convex subset of $X ; I_{i}: \mathcal{B} \rightarrow$ $X, i=1,2, \ldots, m$ are suitable mappings satisfying some conditions which will be specified later, and the symbol $\Delta x(t)$ represents the jump of the function $x$ at $t$, which is defined by $\Delta x(t)=x\left(t^{+}\right)-x\left(t^{-}\right)$, where $x\left(t_{i}^{+}\right)$and $x\left(t_{i}^{-}\right)$represent the right and left limits of $x(t)$ at $t=t_{i}$. Finally $2^{X}$ denotes the family of nonempty subsets of $X$.

The theory of impulsive evolution equations, as well as inclusions, has been proven to be applicable to problems arising in mechanics, electrical engineering, medicine, biology, ecology, population dynamics, etc., and it has been extensively investigated in the

(c) 2015 Lin et al. This article is distributed under the terms of the Creative Commons Attribution 4.0 International License (http://creativecommons.org/licenses/by/4.0/), which permits unrestricted use, distribution, and reproduction in any medium, provided you give appropriate credit to the original author(s) and the source, provide a link to the Creative Commons license, and indicate if changes were made. 
last several decades. For these considerable developments in this topic, one may refer to the monographs of Bainov and Simeonov [2], Benchohra et al. [3], Haddad et al. [4], Lakshmikantham et al. [5], Samoilenko and Perestyuk [6], and [7-9] and the references therein. On the other hand, a partial neutral differential equation with unbounded delay lies in problems from various fields such as macroeconomic model including dynamics of income or value of capital stock (see the book of Chukwu [10]), the theory of viscoelastic fluid (see [11, 12]), and the theory of heat flow in materials with memory. For example, in [13-15], the authors studied the following system:

$$
\begin{aligned}
& \frac{d u}{d t}\left[u(t, x)+\int_{-\infty}^{t} k_{1}(t-s) u(s, x) d s\right] \\
& \quad=c \Delta u(t, x)+\int_{-\infty}^{t} k_{2}(t-s) u(s, x) d s, \quad t \geq 0, \\
& u(t, x)=0, \quad x \in \partial \Omega,
\end{aligned}
$$

where $\Omega \subset \mathbb{R}^{n}$ is open, bounded, and with smooth boundary; $(t, x) \in[0, \infty) \times \Omega, u(t, x)$ represents the temperature in $x$ at the time $t ; c$ is a physical constant and $k_{i}: \mathbb{R} \rightarrow \mathbb{R}$, $i=1,2$, are the internal energy and the heat flux relaxation, respectively. It is clear that this equation can be modeled by system (1.1), and this serves as the physical motivation to study (1.1). We point out that the theory of impulsive neutral evolution differential equations or inclusions with infinite delay assuming axioms introduced by Hale and Kato has been the subject of many papers; see for example [16-20], and many others.

There are several works reporting existence results of mild solutions for impulsive neutral evolution systems with infinite delay similar to (1.1); see for example [19, 20]. In these works, the authors impose compactness on the operator family generated by $A(t)$. However, this condition is too severe in application, which serves as the main motivation of this work. In the present article, we give an existence result for the problem (1.1) without any compactness assumption by using a method based on a fixed point principle regarding a condensing mapping with respect to some nonsingular measure of noncompactness. The approach we use here is much inspired by [21]. The rest of this work is arranged as follows: In Section 2, we recall briefly some basic definitions and preliminary facts which will be used throughout the following sections. In Section 3, we prove the existence of mild solutions problem (1.1), and in the last section an example is addressed to illustrate the obtained abstract result.

\section{Preliminaries}

We introduce certain notations which will be used throughout the article. In this paper, $(X,\|\cdot\|)$ is a Banach space and the linear part $A(t)$ of equation (1.1) are operators in $X$, with domain $D(A(t))=D$ for all $t \in[0, T]$ and dense in $X$ and let $\{U(t, s)\}_{0 \leq s \leq t \leq T} \subset L(X)$ be the evolution family generated by $\{A(t): t \in[0, T]\}$.

Definition 2.1 A family of linear operators $\{U(t, s)\}_{0 \leq s \leq t \leq T} \subset L(X)$ is called an evolution family of operators generated by $\{A(t): t \in[0, T]\}$ if the following conditions hold:

(i) $U(s, s)=I$,

(ii) $U(t, r) U(r, s)=U(t, s)$ for $0 \leq s \leq r \leq t \leq T$,

(iii) $(t, s) \rightarrow U(t, s)$ is strongly continuous for $0 \leq s \leq t \leq T$,

(iv) the function $(s, t] \rightarrow L(X)$ is differentiable with $\frac{\partial}{\partial t} U(t, s)=A(t) U(t, s)$. 
In the following, we put $\Delta:=\{(t, s): 0 \leq s<t \leq T\}$, and

$$
M_{0}:=\sup _{(t, s) \in \Delta}\|U(t, s)\|_{L(X)} .
$$

Let

$$
\begin{aligned}
\mathcal{P C}([0, T], X)= & \left\{x:[0 ; T] \rightarrow X: x(t) \text { be continuous at } t \neq t_{i},\right. \\
& \text { left continuous at } t=t_{i}, \\
& \text { and the right limit } \left.u\left(t_{i}^{+}\right) \text {exists for } i=1,2, \ldots, m\right\} .
\end{aligned}
$$

Evidently $\mathcal{P C}(0, T ; X)$ is a Banach space with the norm

$$
\|x\|_{\mathcal{P C}}=\sup _{t \in[0, T]}\|x(t)\| .
$$

We assume that the state space $\left(\mathcal{B},\|\cdot\|_{\mathcal{B}}\right)$ is a semi-norm linear space of functions mapping $(-\infty, 0]$ into $X$, and satisfying the following axiom first introduced by Hale and Kato in [1]:

(A) If $T>0$ and $x:(-\infty, T] \rightarrow X$ satisfies that $x_{0} \in \mathcal{B}$ and $\left.x\right|_{[0, T]} \in \mathcal{P C}(0, T ; X)$, then for every $t$ in $[0, T]$ the following conditions hold:

(i) $x_{t} \in \mathcal{B}$

(ii) $|x(t)| \leq H\left\|x_{t}\right\|_{\mathcal{B}}$,

(iii) $\left\|x_{t}\right\|_{\mathcal{B}} \leq K(t) \sup _{0 \leq s \leq t}\|x(s)\|+M(t)\left\|x_{0}\right\|_{\mathcal{B}}$,

where $H$ is a constant, $K: \mathbb{R}^{+} \rightarrow \mathbb{R}^{+}$is continuous and $M: \mathbb{R}^{+} \rightarrow \mathbb{R}^{+}$is locally bounded;

$H, K, M$ are independent of $x(\cdot)$.

(B) The space $\mathcal{B}$ is complete.

Let $\mathcal{E}$ be the space

$$
\left\{x:(-\infty, T] \rightarrow X \mid x_{0} \in \mathcal{B} \text { and }\left.x\right|_{[0, T]} \in \mathcal{P C}(0, T ; X)\right\}
$$

with the semi-norm

$$
\|x\|_{\mathcal{E}}=\left\|x_{0}\right\|_{\mathcal{B}}+\left\|\left.x\right|_{[0, T]}\right\| \mathcal{P C} .
$$

Definition 2.2 Let $X$ be a Banach space, $2^{X}$ denote the collection of all nonempty subsets of $X$, and $(\mathcal{A}, \geq)$ a partially ordered set. A function $\mu: 2^{X} \rightarrow \mathcal{A}$ is called a measure of noncompactness in $X$ if

$$
\mu(\overline{\mathrm{co}} \Omega)=\mu(\Omega), \quad \Omega \in 2^{X},
$$

where $\overline{\mathrm{co}} \Omega$ is the closure of convex hull of $\Omega$. A measure of noncompactness $\mu$ is called

(i) monotone, if for each $\Omega_{1}, \Omega_{2} \in 2^{X}$ such that $\Omega_{1} \subset \Omega_{2}$, we have $\mu\left(\Omega_{1}\right) \leq \mu\left(\Omega_{2}\right)$;

(ii) nonsingular, if $\mu(\{a\} \cup \Omega)=\mu(\Omega)$ for any $a \in X, \Omega \in 2^{X}$;

(iii) invariant with respect to the union with a compact set, if $\mu(K \cup \Omega)=\mu(\Omega)$ for every relatively compact set $K \subset X$ and $\Omega \in 2^{X}$. 
Moreover, if $\mathcal{A}$ is a cone in a normed space, we say that $\mu$ is

(iv) algebraically semi-additive, if $\mu\left(\Omega_{1} \cup \Omega_{2}\right) \leq \mu\left(\Omega_{1}\right)+\mu\left(\Omega_{2}\right)$ for any $\Omega_{1}, \Omega_{2} \in 2^{X}$;

(v) regular, if $\mu(\Omega)=0$ is equivalent to the relative compactness of $\Omega$.

The so-called Hausdorff measure of noncompactness, defined by

$$
\chi(\Omega):=\inf \{\varepsilon: \Omega \text { has a finite } \varepsilon \text { net }\}
$$

satisfies all the above properties. For the main result of the present paper, we introduce two measure of noncompactness on the space $\mathcal{P C}([0, T], X)$ of continuous functions on interval $[0, T]$ taking values in $X$.

(i) For each $\Omega \subset \mathcal{P C}([0, T], X)$, the damped modulus of fiber noncompactness of $\Omega$ is defined by

$$
\gamma(\Omega)=\sup _{t \in[0, T]} e^{-L t} \chi(\Omega(t))
$$

where $L$ is a nonnegative constant, $\chi$ is the Hausdorff measure of noncompactness on $X$ and $\Omega(t)=\{\omega(t): \omega \in \Omega\}$.

(ii) For each $\Omega \subset \mathcal{P C}([0, T], X)$, the modulus of equicontinuity of $\Omega$ is defined by

$$
\bmod _{C}(\Omega)=\lim _{\delta \rightarrow 0} \sup _{\omega \in \Omega} \max _{\left|t_{1}-t_{2}\right|<\delta}\left\|\omega\left(t_{1}\right)-\omega\left(t_{2}\right)\right\| .
$$

Now, consider the function $v: 2^{\mathcal{P C}([0, T], X)} \rightarrow[0, \infty] \times[0, \infty]$ given by

$$
v(\Omega)=\max _{S \in D(\Omega)}\left(\gamma(S), \bmod _{C}(S)\right)
$$

where $D(\Omega)$ is the collection of all denumerable subsets of $\Omega$ and the maximum is taken in the sense of the partial order in the cone $[0, \infty] \times[0, \infty]$. It is well known that $v$ is a measure of noncompactness in the space $\mathcal{P C}([0, T], X)$, which satisfies all properties in Definition 2.2 and the maximum in (2.1) is attained in $D(\Omega)$ (see [22], Example 2.1.3 for details).

In the following, $X$ will be a separable Banach space and we shall use the notations:

$$
\begin{aligned}
& P_{c l}(X):=\left\{Y \in 2^{X}: Y \text { is closed }\right\}, \\
& P_{b}(X):=\left\{Y \in 2^{X}: Y \text { is bounded }\right\}, \\
& P_{c}(X):=\left\{Y \in 2^{X}: Y \text { is convex }\right\}, \\
& P_{k}(X):=\left\{Y \in 2^{X}: Y \text { is compact }\right\} .
\end{aligned}
$$

A multimap $\mathcal{F}:[0, T] \rightarrow P_{k}(X)$ is said to be measurable, if $\mathcal{F}^{-1}(W)$ is measurable for every open subset $W$ of $X$, where $\mathcal{F}^{-1}(W):=\{w \in[0, T]: \mathcal{F}(w) \cap W \neq \varnothing\}$. A multimap $\mathcal{F}(\cdot)$ is integrably bounded if and only if $\mathcal{F}(\cdot)$ is measurable and $\|\mathcal{F}(\cdot)\| \in L^{1}([0, T], \mathbb{R})$, where $\|\mathcal{F}(t)\|=\sup _{f \in F(t)}\|f\|$. For any multifunction $\mathcal{F}:[0, T] \rightarrow 2^{X}$ and for $1 \leq p \leq \infty$ we denote $S_{\mathcal{F}}^{p}$, the set of all selectors of $G(\cdot, x$.), which belong to the Lebesgue-Bochner space $L^{p}([0, T], X)$, i.e.,

$$
S_{\mathcal{F}}^{p}=\left\{f(\cdot) \in L^{p}([0, T], X): f(t) \in \mathcal{F}(t) \text { a.e. } t \in[0, T]\right\} .
$$


If $S_{\mathcal{F}}^{p}$ is nonempty and $F(\cdot)$ is closed valued, then it is well known that $S_{\mathcal{F}}^{p}$ is a closed subset of $L^{p}([0, T], X)(1 \leq p \leq \infty)$. The following result, which is adapted from [23], is crucial to our main result.

Lemma 2.1 If $\mathcal{F}:[0, T] \rightarrow P_{w k c}(X)$ is integrably bounded, then $S_{\mathcal{F}}^{1}$ is nonempty, convex and w-compact in $L^{1}([0, T], X)$.

Denote by $\mathcal{W}: L^{1}([0, T], X) \rightarrow \mathcal{P C}([0, T], X)$ the operator

$$
\mathcal{W} \psi(t)=\int_{0}^{t} U(t, s) \psi(s) d s
$$

This is the so-called generalized Cauchy operator and it is well known (see [22]) that it satisfies the following properties:

$(\mathcal{W} 1) \mathcal{W}$ sends each bounded set to an equicontinuous one;

$(\mathcal{W} 2)$ there exists a constant $C>0$ such that

$$
\left\|\mathcal{W}\left(\psi_{1}\right)(t)-\mathcal{W}\left(\psi_{2}\right)(t)\right\| \leq C \int_{0}^{t}\left\|\psi_{1}(s)-\psi_{2}(s)\right\| d s
$$

for all $\psi_{1}, \psi_{2} \in L^{1}([0, T], X), t \in[0, T]$;

$(\mathcal{W} 3)$ for each compact set $K \subset X$ and sequence $\left\{\psi_{n}\right\} \subset L^{1}([0, T], X)$ such that $\left\{\psi_{n}(t)\right\} \subset K$ for a.e. $t \in[0, T]$, the weak convergence $\psi_{n} \rightarrow \psi_{0}$ implies $\mathcal{W}\left(\psi_{n}\right) \rightarrow \mathcal{W}\left(\psi_{0}\right)$ strongly in $\mathcal{P C}([0, T], X)$.

The following lemma, which also comes from [22], is useful in the proof of our main result.

Lemma 2.2 Let $\left\{\psi_{n}\right\}$ be an integrably bounded sequence in $L^{1}([0, T], X)$, i.e.,

$$
\left\|\psi_{n}(t)\right\| \leq \eta(t) \text { for a.e. } t \in[0, T]
$$

where $\eta \in L^{1}([0, T])$. If $Q$ satisfies $(\mathcal{W} 2),(\mathcal{W} 3)$ and there exists $q \in L^{1}([0, T])$ such that

$$
\chi\left(\left\{\psi_{n}(t)\right\}_{n=1}^{\infty}\right) \leq q(t) \text { for a.e. } t \in[0, T]
$$

then

$$
\chi\left(\left\{Q\left(\psi_{n}\right)(t)\right\}_{n=1}^{\infty}\right) \leq 2 C \int_{0}^{t} q(s) d s
$$

for each $t \in[0, T]$, where $C>0$ is the constant given in condition $(\mathcal{W} 2)$.

Definition 2.3 A multimap $\mathcal{F}: X \rightarrow P_{k}(X)$ is said to be condensing with respect to a measure of noncompactness $\mu$ or $\mu$-condensing if for every bounded set $\Omega \subset X$, the relation

$$
\mu(\mathcal{F}(\Omega)) \geq \mu(\Omega)
$$

implies the relative compactness of $\Omega$. 
The following fixed point principle can be found in [22].

Lemma 2.3 If $U$ is a closed convex subset of a Banach space $X$ and $\Phi: U \rightarrow P_{c v, k}(X)$ is a closed $\mu$-condensing multimap, where $\mu$ is a nonsingular measure of noncompactness defined on the subsets of $U$, then $\Phi$ has a fixed point.

\section{Existence result}

The system (1.1) has the following equivalent form:

$$
\left\{\begin{array}{l}
\frac{d}{d t}\left[x(t)+F\left(t, x_{t}\right)\right] \\
\quad \in A(t)\left[x(t)+F\left(t, x_{t}\right)\right]-A(t) F\left(t, x_{t}\right)+G\left(t, x_{t}\right), \quad t \in[0, T], t \neq t_{i}, \\
\Delta x\left(t_{i}\right)=I_{i}\left(x_{t_{i}}\right), \quad i=1,2, \ldots, m, \\
x_{0}=\varphi \in \mathcal{B} .
\end{array}\right.
$$

Let $x:(-\infty, T] \rightarrow X$ be a function such that $x, x^{\prime} \in \mathcal{E}$. If $x$ is a solution of $(1.1)$, then from the semi-group theory (see [24], Sections 4.3 and 4.4), there exist functions $f, g \in L^{1}([0, T], X)$ with $f \in F\left(t, x_{t}\right), g \in G\left(t, x_{t}\right)$ for a.e. $t \in[0, T]$ such that

$$
\begin{aligned}
x(t)+f(t)= & U(t, 0)[\varphi(0)+f(0)]-\int_{0}^{t} A(s) U(t, s) f(s) d s \\
& +\int_{0}^{t} U(t, s) g(s) d s .
\end{aligned}
$$

This implies that

$$
\begin{aligned}
x\left(t_{1}^{-}\right)+f\left(t_{1}\right)= & U\left(t_{1}, 0\right)[\varphi(0)+f(0)]-\int_{0}^{t_{1}} A(s) U\left(t_{1}, s\right) f(s) d s \\
& +\int_{0}^{t_{1}} U\left(t_{1}, s\right) g(s) d s,
\end{aligned}
$$

and since $x\left(t_{1}^{+}\right)=x\left(t_{1}^{-}\right)+I_{1}\left(x_{t_{1}}\right)$, we have, for $t \in\left(t_{1}, t_{2}\right)$,

$$
\begin{aligned}
x(t)+f(t)= & U\left(t, t_{1}\right)\left[x\left(t_{1}^{+}\right)+f\left(t_{1}\right)\right]-\int_{t_{1}}^{t} A(s) U(t, s) f(s) d s \\
& +\int_{t_{1}}^{t} U(t, s) g(s) d s \\
= & U\left(t, t_{1}\right)\left[x\left(t_{1}^{-}\right)+f\left(t_{1}\right)+I_{1}\left(x_{t_{1}}\right)\right]-\int_{t_{1}}^{t} A(s) U(t, s) f(s) d s \\
& +\int_{t_{1}}^{t} U(t, s) g(s) d s \\
= & U\left(t, t_{1}\right)\left[U\left(t_{1}, 0\right)(\varphi(0)+f(0))-\int_{0}^{t_{1}} A(s) U\left(t_{1}, s\right) f(s) d s\right. \\
& \left.+\int_{0}^{t_{1}} U\left(t_{1}, s\right) g(s) d s+I_{1}\left(x_{t_{1}}\right)\right]-\int_{t_{1}}^{t} A(s) U(t, s) f(s) d s \\
& +\int_{t_{1}}^{t} U(t, s) g(s) d s
\end{aligned}
$$




$$
\begin{gathered}
=U(t, 0)[\varphi(0)+f(0)]-\int_{0}^{t} A(s) U(t, s) f(s) d s \\
+\int_{0}^{t} U(t, s) g(s) d s+U\left(t, t_{1}\right) I_{1}\left(x_{t_{1}}\right) .
\end{gathered}
$$

Reiterating these procedures, we can prove that

$$
\begin{aligned}
x(t)= & U(t, 0)[\varphi(0)+f(0)]-f(t)-\int_{0}^{t} A(s) U(t, s) f(s) d s \\
& +\int_{0}^{t} U(t, s) g(s) d s+\sum_{i=1}^{m} U\left(t, t_{i}\right) I_{i}\left(x_{t_{i}}\right), \quad t \in[0, T] .
\end{aligned}
$$

This expression motivates the following definition.

Definition 3.1 We say that a function $x \in \mathcal{E}$ is a mild solution of the system (1.1) if $x_{0}=\varphi$, $x_{t} \in \mathcal{B}$ for every $t \in[0, T], \Delta x\left(t_{i}\right)=I_{i}\left(x_{t_{i}}\right), i=1,2, \ldots, m$, the function $s \mapsto A(s) U(t, s) F\left(s, x_{s}\right)$ is Bochner integrable on $[0, T]$, and the impulsive integral inclusion

$$
\begin{gathered}
x(t) \in U(t, 0)[\varphi(0)+F(0, \varphi)]-F\left(t, x_{t}\right)-\int_{0}^{t} A(s) U(t, s) F\left(s, x_{s}\right) d s \\
+\int_{0}^{t} U(t, s) G\left(s, x_{s}\right) d s+\sum_{i=1}^{m} U\left(t, t_{i}\right) I_{i}\left(x_{t_{i}}\right), \quad t \in[0, T],
\end{gathered}
$$

is satisfied.

In order to study system (1.1), we impose the following assumptions.

(H1) There is a Banach space $\left(Y,\|\cdot\|_{Y}\right)$ continuously embedded in $X$ such that the function $s \mapsto A(s) U(t, s)$ defined from $[0, T]$ into $L(Y, X)$ is strongly measurable and there exists a function $\mathcal{H} \in L^{1}([0, T])$ for which the following holds:

$$
\|A(s) U(t, s)\|_{L(Y, X)} \leq \mathcal{H}(t-s) \quad \text { for } 0 \leq s<t \leq T
$$

(H2) The function $F$ satisfies

(i) $F$ is $Y$-valued and $F:[0, T] \times \mathcal{B} \rightarrow Y$ is continuous;

(ii) $F:[0, T] \times \mathcal{B} \rightarrow X$ is completely continuous;

(iii) there exists $L_{F}>0$ such that

$$
\left\|F\left(t, \varphi_{1}\right)-F\left(s, \varphi_{2}\right)\right\|_{Y} \leq L_{F}\left(|t-s|+\left\|\varphi_{1}-\varphi_{2}\right\|_{\mathcal{B}}\right)
$$

for any $t, s \in[0, T]$ and $\varphi_{1}, \varphi_{2} \in \mathcal{B}$.

(H3) The multimap $G:[0, T] \times \mathcal{B} \rightarrow 2^{X}$ satisfies the Carathéodory condition (see [25], p.298), i.e., for each $\varphi \in \mathcal{B}, G(\cdot, \varphi)$ has a strongly measurable selection, and for a.e. $t \in[0, T], G(t, \cdot): \mathcal{B} \rightarrow P_{c, k}(X)$ is upper semi-continuous. Moreover, there exists a function $\alpha \in L^{1}\left([0, T], \mathbb{R}^{+}\right)$such that

$$
\|G(t, \varphi)\| \leq \alpha(t)\left(1+\|\varphi\|_{\mathcal{B}}\right) \quad \text { for a.e. } t \in[0, T]
$$

where $\|G(t, \varphi)\|:=\sup _{g \in G(, \varphi)}\|g(t)\|$. 
(H4) For each $V \subset \mathcal{E}$, let

$$
V_{\mathcal{B}}:=\left\{\phi \in \mathcal{B}: \phi=x_{t} \text { for some } x \in V \text { and } t \in[0, T]\right\}
$$

Then there exists a function $\beta:[0, T] \in L^{1}\left([0, T], \mathbb{R}^{+}\right)$such that, for all bounded set $V \subset \mathcal{E}$,

$$
\chi\left(\bigcup_{\phi \in V_{\mathcal{B}}} G(t, \phi)\right) \leq \beta(t) \chi\left(V_{\mathcal{P C}}\right) \quad \text { for a.e. } t \in[0, t]
$$

where $V_{\mathcal{P C}}:=\{x(t) ; x \in V, t \in[0, T]\}$ and $\chi$ is the Hausdorff measure of noncompactness.

Remark 3.1 It is clear that assumption (H4) is fulfilled if $G$ is compact in its second argument, i.e., for each $t \in[0, T]$ and bounded $\Omega \subset \mathcal{B}$, the set $G(t, \Omega)=\bigcup_{\omega \in \Omega} G(t, \omega)$ is relatively compact in $X$.

(H5) The function $I_{i}: \mathcal{B} \rightarrow X$ is continuous and there are positive constants $L_{i}$, $i=1,2, \ldots, m$, such that

$$
\left\|I_{i}\left(\phi_{1}\right)-I_{i}\left(\phi_{2}\right)\right\| \leq L_{i}\left\|\phi_{1}-\phi_{2}\right\|_{\mathcal{B}}
$$

for $\phi_{1}, \phi_{2} \in \mathcal{B}$ and $i=1,2, \ldots, m$.

Note that the assumptions (H1) and (H2) are linked to the integrability of the function $s \rightarrow A(s) U(t, s) f\left(s, x_{s}\right)$. In general, we observe that, except in trivial cases, the operator function $s \rightarrow A(s) U(t, s)$ is not integrable over [0,T]; for an interpretation of this observation, we refer the reader to the article [18]. We are now in a position to state and prove the main result of this section.

Theorem 3.1 Suppose that the hypotheses (H1)-(H5) are satisfied. Then the system (1.1) has at least one mild solution provided

$$
\tilde{K}\left[(1+\tilde{\mathcal{H}}) L_{F}+M_{0}\left(\|\alpha\|_{L^{1}}+\sum_{i=1}^{m} L_{i}\right)\right]<1,
$$

where $\tilde{\mathcal{H}}:=\|\mathcal{H}\|_{L^{1}([0, T])}$ and $\tilde{K}:=\sup _{0 \leq t \leq T}|K(t)|$.

Proof We introduce the multioperator $\Gamma: \mathcal{P C}([0, T], X) \rightarrow 2^{\mathcal{P C}([0, T], X)}$ by

$$
\begin{aligned}
(\Gamma x)(t):= & \left\{U(t, 0)[\varphi(0)+F(t, \varphi)]-F\left(t, \bar{x}_{t}\right)+\int_{0}^{t} A(s) U(t, s) F\left(s, \bar{x}_{s}\right) d s\right. \\
& \left.+\int_{0}^{t} U(t, s) g(s) d s+\sum_{i=1}^{m} U\left(t, t_{i}\right) I_{i}\left(\bar{x}_{t_{i}}\right) ; g \in S_{G(\cdot, \bar{x} .)}^{1}, t \in[0, T]\right\},
\end{aligned}
$$

where for every $x \in \mathcal{P C}([0, T], X), \bar{x}$ denotes the extension of $x$ to $\mathcal{E}$ given by

$$
\bar{x}(t)= \begin{cases}x(t), & t \in[0, T], \\ \varphi(t), & t \leq 0 .\end{cases}
$$


It is readily seen that if $x \in \operatorname{Fix}(\Gamma):=\{x \in \mathcal{P C}([0, T], X): x \in \Gamma(x)\}$, then $\bar{x}$ is a mild solution of the system (1.1). It therefore suffices to show that the set Fix $(\Gamma)$ is nonempty, and the proof is divided into several steps.

Step 1 . It is already seen that $\Gamma$ has convex values, using the hypotheses that the multimap $G$ has convex values.

Step 2. To see that $\Gamma$ has closed graph, let $\left\{x^{n}\right\}_{n=1}^{\infty}$ be a sequence in $\mathcal{P C}([0, T], X)$ with $x^{n} \rightarrow x \in \mathcal{P C}([0, T], X)$. Then by axiom (A)(iii),

$$
\begin{aligned}
\left\|\bar{x}_{t}^{n}-\bar{x}_{t}\right\|_{\mathcal{B}} & \leq K(t) \sup _{s \in[0, T]}\left\|x^{n}(s)-x(s)\right\|+M(t)\left\|\bar{x}_{0}^{n}-\bar{x}_{0}\right\|_{\mathcal{B}} \\
& =K(t) \sup _{s \in[0, T]}\left\|x^{n}(s)-x(s)\right\| \\
& \rightarrow 0 \quad \text { as } n \rightarrow \infty .
\end{aligned}
$$

Now, for each $n \in \mathbb{N}$, choose $y^{n} \in \Gamma\left(x_{n}\right)$. Then by (3.2), for each $n \in \mathbb{N}$, the mapping $t \mapsto G\left(t, \bar{x}_{t}^{n}\right)$ admits a selector $g_{n}$ such that

$$
\begin{aligned}
y^{n}(t):= & U(t, 0)[\varphi(0)+F(t, \varphi)]-F\left(t, \bar{x}_{t}^{n}\right)+\int_{0}^{t} A(s) U(t, s) F\left(s, \bar{x}_{s}^{n}\right) d s \\
& +\int_{0}^{t} U(t, s) g_{n}(s) d s+\sum_{i=1}^{m} U\left(t, t_{i}\right) I_{i}\left(\bar{x}_{t_{i}}^{n}\right), \quad t \in[0, T] .
\end{aligned}
$$

Let

$$
W(t)=\overline{\mathrm{co}} \bigcup_{n \geq 1} G\left(t, \bar{x}_{t}^{n}\right)
$$

Invoking Theorem 7.4.2 of [26], p.90 and (H3), we have $W(t) \in P_{w k c}(X)$. Again by (H3), we have

$$
\|W(t)\|=\sup _{w \in W(t)}\|w\| \leq \alpha(t)(1+B)
$$

yielding the result that $W(\cdot)$ is integrably bounded, where $B=\sup _{n \geq 1}\left\|\bar{x}^{n}\right\|_{\mathcal{B}}$, and hence by Lemma 2.1, we see that $S_{W}^{1}$ is weakly compact in $L^{1}([0, T], X)$. We thus may assume, by passing to a subsequence if necessary, that

$$
g_{n} \rightarrow^{w} g \text { in } L^{1}([0, T], X) .
$$

Moreover, it follows by Theorem 3.1 of [27] that

$$
g(t) \in \overline{\mathrm{co}}\left\{w-\varlimsup_{n}\left\{g_{n}(t)\right\}\right\} \subset \overline{\mathrm{co}}\left\{w-\varlimsup_{n} G\left(t, \bar{x}_{t}^{n}\right)\right\} \subset G\left(t, \bar{x}_{t}\right) \quad \text { a.e. on }[0, T],
$$

where the last inclusion is guaranteed by (H3). Now,

$$
\begin{aligned}
y^{n}(t)= & U(t, 0)[\varphi(0)+F(t, \varphi)]-F\left(t, \bar{x}_{t}^{n}\right)-\int_{0}^{t} A(s) U(t, s) F\left(s, \bar{x}_{s}^{n}\right) d s \\
& +\int_{0}^{t} U(t, s) g_{n}(s) d s+\sum_{i=1}^{m} U\left(t, t_{i}\right) I_{i}\left(\bar{x}_{t_{i}}^{n}\right), \quad t \in[0, T]
\end{aligned}
$$




$$
\begin{aligned}
= & U(t, 0)[\varphi(0)+F(t, \varphi)]-F\left(t, \bar{x}_{t}^{n}\right)-\int_{0}^{t} A(s) U(t, s) F\left(s, \bar{x}_{s}^{n}\right) d s \\
& +\mathcal{W} g_{n}(t)+\sum_{i=1}^{m} U\left(t, t_{i}\right) I_{i}\left(\bar{x}_{t_{i}}^{n}\right), \quad t \in[0, T] \\
\rightarrow & U(t, 0)[\varphi(0)+F(t, \varphi)]-F\left(t, \bar{x}_{t}\right)-\int_{0}^{t} A(s) U(t, s) F\left(s, \bar{x}_{s}\right) d s \\
& +\mathcal{W} g(t)+\sum_{i=1}^{m} U\left(t, t_{i}\right) I_{i}\left(\bar{x}_{t_{i}}\right), \quad t \in[0, T],
\end{aligned}
$$

by (3.3), (3.4), ( $\mathcal{W} 3),(\mathrm{H} 1),(\mathrm{H} 2)$, and the dominated convergence theorem. Set

$$
\begin{aligned}
y:= & U(t, 0)[\varphi(0)+F(t, \varphi)]-F\left(t, \bar{x}_{t}\right)-\int_{0}^{t} A(s) U(t, s) F\left(s, \bar{x}_{s}\right) d s \\
& +\mathcal{W} g(t)+\sum_{i=1}^{m} U\left(t, t_{i}\right) I_{i}\left(\bar{x}_{t_{i}}\right) .
\end{aligned}
$$

In view of (3.5), we see that

$$
y \in \Gamma(x),
$$

and hence $\Gamma$ has a closed graph. By a similar argument, we find that $\Gamma$ has compact values.

Step 3. We now prove that $\Gamma$ is $\nu$-condensing. To this aim, consider a bounded set $\Omega \subset$ $\mathcal{P C}([0, T], X)$ such that

$$
v(\Gamma(\Omega)) \geq v(\Omega) .
$$

We will show that $\Omega$ is relatively compact in $\mathcal{P C}([0, T], X)$. In fact, there exists, by the definition of $v$, a sequence $\left\{z^{n}\right\}_{n=1}^{\infty}$ which reaches the maximum, i.e.,

$$
v(\Gamma(\Omega))=\left(\gamma\left(\left\{z^{n}\right\}_{n=1}^{\infty}\right), \bmod _{C}\left(\left\{z^{n}\right\}_{n=1}^{\infty}\right)\right) .
$$

Choose $\left\{x^{n}\right\}_{n=1}^{\infty} \subset \Omega$ so that for each $n \in \mathbb{N}, z_{n} \in \Gamma\left(x_{n}\right)$. Then

$$
\begin{aligned}
z^{n}(t)= & U(t, 0)[\varphi(0)+F(t, \varphi)]-F\left(t, \bar{x}_{t}^{n}\right)-\int_{0}^{t} A(s) U(t, s) F\left(s, \bar{x}_{s}^{n}\right) d s \\
& +\int_{0}^{t} U(t, s) g_{n}(s) d s+\sum_{i=1}^{m} U\left(t, t_{i}\right) I_{i}\left(\bar{x}_{t_{i}}^{n}\right), \quad t \in[0, T],
\end{aligned}
$$

where $g_{n} \in S_{G\left(\cdot, \bar{x}^{n}\right)}^{1}$, so that

$$
\gamma\left(\left\{z^{n}\right\}_{n=1}^{\infty}\right)=\gamma\left(\left\{\mathcal{W} g_{n}\right\}_{n=1}^{\infty}\right)
$$

since $F:[0, T] \times \mathcal{B} \rightarrow X$ is completely continuous by (H2). Now, let $t \in[0, T]$ and it follows by $(\mathrm{H} 4)$ that

$$
\begin{aligned}
\chi\left(\left\{g_{n}(s)\right\}_{n=1}^{\infty}\right) & \leq \beta(s) \chi\left(\left\{x^{n}(\tau)\right\}_{n=1}^{\infty}\right) \leq \beta(s) e^{L s} \sup _{0 \leq \tau \leq T} e^{-L \tau} \chi\left(\left\{x^{n}(\tau)\right\}_{n=1}^{\infty}\right) \\
& =\beta(s) e^{L s} \gamma\left(\left\{x^{n}\right\}_{n=1}^{\infty}\right)
\end{aligned}
$$


for all $s \in[0, t]$. Now, we apply Lemma 2.2 and obtain

$$
\chi\left(\left\{\mathcal{W} g_{n}(t)\right\}_{n=1}^{\infty}\right) \leq 2 C\left(\int_{0}^{t} \beta(s) e^{L s} d s\right) \gamma\left(\left\{x^{n}\right\}_{n=1}^{\infty}\right),
$$

which implies

$$
e^{-L t} \chi\left(\left\{\mathcal{W} g_{n}(t)\right\}_{n=1}^{\infty}\right) \leq 2 C\left(\int_{0}^{t} \beta(s) e^{-L(t-s)} d s\right) \gamma\left(\left\{x^{n}\right\}_{n=1}^{\infty}\right),
$$

whence, in view of (3.6),

$$
\gamma\left(\left\{x^{n}\right\}_{n=1}^{\infty}\right) \leq \gamma\left(\left\{z^{n}\right\}_{n=1}^{\infty}\right) \leq \zeta \gamma\left(\left\{x^{n}\right\}_{n=1}^{\infty}\right) .
$$

Here

$$
\zeta:=2 C \sup _{t \in[0, T]} \int_{0}^{t} e^{-L(t-s)} \beta(s) d s .
$$

Now, choose the constant $L>0$ in the definition of $\gamma$ so that

$$
\zeta:=2 C \sup _{t \in[0, T]} \int_{0}^{t} e^{-L(t-s)} \beta(s) d s<1,
$$

and we thus combine (3.7) and (3.8) to conclude

$$
\gamma\left(\left\{z^{n}\right\}_{n=1}^{\infty}\right)=0 .
$$

On the other hand, it is evident from (H3) that $\left\{g_{n}\right\}$ is a bounded sequence in $L^{1}([0, T], X)$. Then the property $(\mathcal{W} 1)$ ensures that $\left\{\mathcal{W} g_{n}\right\}$ is equicontinuous in $\mathcal{P C}([0, T], X)$ and hence

$$
\bmod _{C}\left(\left\{z^{n}\right\}_{n=1}^{\infty}\right)=\bmod _{C}\left(\left\{\mathcal{W} g_{n}\right\}_{n=1}^{\infty}\right)=0
$$

Consequently,

$$
v(\Omega)=(0,0)
$$

and therefore, the regularity of $v$ guarantees the relative compactness of $\Omega$.

Step 4. To use Lemma 2.3, we shall demonstrate that the solution set belongs to $a$ priori bounded set in $\mathcal{P C}([0, T], X)$. Indeed, if this were not the case, the for each $n \in \mathbb{N}$, there is an $x^{n} \in \mathcal{P C}([0, T], X)$ with $\left\|x^{n}\right\| \leq n$ but $\left\|\Gamma x^{n}\right\|>n$. Now, for each $n \in \mathbb{N}$, let $g_{n} \in S_{G\left(\cdot, \bar{x}^{n}\right)}^{1}$ and then for every $t \in[0, T]$ we have

$$
\begin{aligned}
\left\|\Gamma x^{n}(t)\right\|= & \| U(t, 0)[\varphi(0)+F(t, \varphi)]-F\left(t, \bar{x}_{t}^{n}\right)-\int_{0}^{t} A(s) U(t, s) F\left(s, \bar{x}_{s}^{n}\right) d s \\
& +\int_{0}^{t} U(t, s) g(s) d s+\sum_{i=1}^{m} U\left(t, t_{i}\right) I_{i}\left(\bar{x}_{t_{i}}^{n}\right) \| .
\end{aligned}
$$


Now, by assumptions (H1), (H2), (H3), and axiom (A)(iii), we have the following estimates:

$$
\begin{aligned}
& \|U(t, 0)[\varphi(0)+F(t, \varphi)]\| \\
& \leq M_{0}\left(\|\varphi(0)\|+\|F(t, \varphi)\|_{Y}\right) \\
& \leq M_{0}\left(\|\varphi(0)\|+\|F(t, \varphi)-F(0,0)\|_{Y}+\|F(0,0)\|_{Y}\right) \\
& \leq M_{0}\left[\|\varphi(0)\|+L_{F}\left(T+\|\varphi\|_{\mathcal{B}}\right)+\|F(0,0)\|_{Y}\right], \\
& \left\|F\left(t, \bar{x}_{t}^{n}\right)\right\| \leq\left\|F\left(t, \bar{x}_{t}^{n}\right)\right\|_{Y} \leq\left\|F\left(t, \bar{x}_{t}^{n}\right)-F(0,0)\right\|_{Y}+\|F(0,0)\|_{Y} \\
& \leq L_{F}\left(t+\left\|\bar{x}_{t}^{n}\right\|_{\mathcal{B}}\right)+\|F(0,0)\|_{Y} \\
& \leq L_{F}\left(t+\tilde{K} \sup _{0 \leq \tau \leq t}\|x(\tau)\|+\tilde{M}\|\varphi\|_{\mathcal{B}}\right)+\|F(0,0)\|_{Y} \\
& =L_{F}\left(T+\tilde{M}\|\varphi\|_{\mathcal{B}}\right)+\|F(0,0)\|_{Y}+L_{F} \tilde{K} \sup _{0 \leq \tau \leq t}\left\|x^{n}(\tau)\right\|, \\
& \left\|\int_{0}^{t} A(s) U(t, s) F\left(s, \bar{x}_{s}^{n}\right) d s\right\| \\
& \leq \int_{0}^{t}\|A(s) U(t, s)\|_{L(Y, X)}\left\|F\left(s, \bar{x}_{s}^{n}\right)\right\|_{Y} d s \\
& \leq\left(\int_{0}^{t} \mathcal{H}(t-s) d s\right)\left[L_{F}\left(t+\tilde{K} \sup _{0 \leq \tau \leq t}\|x(\tau)\|+\tilde{M}\|\varphi\|_{\mathcal{B}}\right)+\|F(0,0)\|_{Y}\right] \\
& \leq \tilde{\mathcal{H}}\left[L_{F}\left(T+\tilde{M}\|\varphi\|_{\mathcal{B}}\right)+\|F(0,0)\|_{Y}\right]+\tilde{\mathcal{H}} L_{F} \tilde{K} \sup _{0 \leq \tau \leq t}\|x(\tau)\|, \\
& \left\|\int_{0}^{t} U(t, s) g(s) d s\right\| \leq M_{0} \int_{0}^{t}\|g(s)\| d s \\
& \leq M_{0} \int_{0}^{t} \alpha(s)\left(1+\left\|\bar{x}_{s}^{n}\right\|_{\mathcal{B}}\right) d s \\
& \leq M_{0}\left(\int_{0}^{t} \alpha(s) d s\right)\left(1+K(s) \sup _{0 \leq \tau \leq s}\left\|x^{n}(\tau)\right\|+M(s)\left\|\bar{x}_{0}^{n}\right\|_{\mathcal{B}}\right) \\
& \leq M_{0}\|\alpha\|_{L^{1}([0, T])}\left(1+\tilde{M}\|\varphi\|_{\mathcal{B}}+\tilde{K} \sup _{0 \leq \tau \leq s}\left\|x^{n}(\tau)\right\|\right),
\end{aligned}
$$

and

$$
\begin{aligned}
& \left\|\sum_{i=1}^{m} U\left(t, t_{i}\right) I_{i}\left(\bar{x}_{t_{i}}^{n}\right)\right\| \\
& \quad \leq \sum_{i=1}^{m}\left\|U\left(t, t_{i}\right)\right\|_{L(X)}\left\|I_{i}\left(\bar{x}_{t_{i}}^{n}\right)\right\| \\
& \quad \leq M_{0} \sum_{i=1}^{m}\left\|I_{i}\left(\bar{x}_{t_{i}}^{n}\right)-I_{i}(0)+I_{i}(0)\right\| \\
& \quad \leq M_{0} \sum_{i=1}^{m}\left(L_{i}\left\|\bar{x}_{t_{i}}^{n}\right\|_{\mathcal{B}}+\left\|I_{i}(0)\right\|\right) \\
& \quad \leq M_{0} \sum_{i=1}^{m}\left(L_{i}\left(\tilde{K} \sup _{0 \leq \tau \leq s}\left\|x^{n}(\tau)\right\|+\tilde{M}\|\varphi\|_{\mathcal{B}}\right)+\left\|I_{i}(0)\right\|\right),
\end{aligned}
$$


where $\tilde{M}:=\sup _{0 \leq t \leq T}|M(t)|$. Thus, it follows by (3.9)-(3.14) and (H5) that

$$
\begin{aligned}
n< & \left\|x^{n}\right\|_{\mathcal{P C}([0, T], X)} \\
\leq & M_{0}\|\varphi(0)\|+(2+\tilde{\mathcal{H}}) L_{F} T+\left(M_{0}+L_{F}+\tilde{\mathcal{H}}\right)\|F(0,0)\|_{Y} \\
& +\left(M_{0} L_{F}+L_{F} \tilde{M}+L_{F} \tilde{\mathcal{H}} \tilde{M}+M_{0} \tilde{M}\|\alpha\|_{L^{1}}\right)\|\varphi\|_{\mathcal{B}} \\
& +M_{0}\|\alpha\|_{L^{1}}+M_{0}\left(m\left\|I_{i}(0)\right\|+\tilde{M}\|\varphi\|_{\mathcal{B}} \sum_{i=1}^{m} L_{i}\right) \\
& +\tilde{K}\left[(1+\tilde{\mathcal{H}}) L_{F}+M_{0}\left(\|\alpha\|_{L^{1}}+\sum_{i=1}^{m} L_{i}\right)\right] n .
\end{aligned}
$$

Divide both sides by $n$ and take the lower limit as $n \rightarrow \infty$; we get

$$
\tilde{K}\left[(1+\tilde{\mathcal{H}}) L_{F}+M_{0}\left(\|\alpha\|_{L^{1}}+\sum_{i=1}^{m} L_{i}\right)\right] \geq 1,
$$

which contradicts (3.1). This completes the proof.

\section{An example}

Let $X:=L^{2}([0,1])$ and denote by $\|\cdot\|$ the usual $L^{2}$-norm of $X$. Let

$$
D:=\left\{\psi \in X: \psi \text { and } \psi^{\prime} \text { are absolutely continuous, } \psi^{\prime \prime} \in X, \psi(0)=\psi(1)=0\right\} .
$$

For each $t \in[0, T]$, define

$$
(A(t) \psi)(\xi)=a(t, \xi) \psi^{\prime \prime}(\xi)+b(t, \xi) \varphi^{\prime}(\xi)+c(t, \xi) \psi(\xi), \quad \xi \in[0,1]
$$

for $\psi \in D(A(t)):=D$, where $a(t, \xi)>0$ for all $t \in[0, T]$ and $\xi \in[0,1]$ and

$$
a, b, c \in C^{\alpha}([0, T], X)
$$

for some $\alpha \in(0,1)$. By [28], Corollary 3.1.21(ii), p.97, $A(t)$ are sectorial operators in $X$, and (4.1) implies that $A(\cdot) \in C^{\alpha}([0, T], L(D, X))$, where $D$ is endowed with the norm $\|\psi\|_{D}:=$ $\sum_{i=0}^{2}\left\|D^{i} \psi\right\|, \psi \in D$. We put

$$
\tilde{A}:=\sup _{s \in[0, T]}\|A(s)\|_{L(D, X)}
$$

Here, for a Banach space $\mathbb{X}$ and an interval $I, C^{\alpha}(I, \mathbb{X})$ stands for the space of Hölder continuous functions $I$ defined as follows:

$$
\begin{aligned}
& C^{\alpha}(I, \mathbb{X})=\left\{f \in C_{b}(I, \mathbb{X}) \mid[f]_{C^{\alpha}(I, \mathbb{X})}=\sup _{t, s \in I, s<t} \frac{\|f(t)-f(s)\|}{(t-s)^{\alpha}}<+\infty\right\}, \\
& \|f\|_{C^{\alpha}(I, \mathbb{X})}=\|f\|_{\infty}+[f]_{C^{\alpha}(I, \mathbb{X})},
\end{aligned}
$$


where $C_{b}(I, \mathbb{X})$ is the space of all bounded and continuous functions on $I$ equipped with sup-norm. It is well known that the parabolic nonautonomous system

$$
\left\{\begin{array}{l}
x^{\prime}(t)=A(t) x(t), \quad t \geq s \\
x(s)=x_{0} \in X
\end{array}\right.
$$

has an associated evolution family $\{U(t, s)\}_{t \geq s}$ on $X$, and we put $M_{0}=\sup _{0 \leq s<t \leq T} \| U(t$, s) $\|_{L(X)}$. We take the phase space

$$
\mathcal{B}=C_{\gamma}=\left\{\phi \in C((-\infty, 0], X): \lim _{\sigma \rightarrow-\infty} e^{\gamma \sigma} \phi(\sigma) \text { exists in } X\right\},
$$

where $\gamma>0$ and set

$$
\|\phi\|_{\mathcal{B}}=\sup _{-\infty<\sigma \leq 0} e^{\gamma \sigma}\|\phi(\sigma)\|, \quad \phi \in C_{\gamma} .
$$

It is well known (see [29], p.14) that $C_{\gamma}$ satisfies the axioms (A) and (B) with

$$
H=1, \quad K(t)=\max \left(1, e^{-\gamma t}\right) \quad \text { and } \quad M(t)=e^{-\gamma t} .
$$

In this section, we consider the following system:

$$
\left\{\begin{array}{l}
\frac{\partial}{\partial t}\left[x(t, \xi)+\int_{-\infty}^{t} \int_{0}^{1} e^{-c_{1}(t-s)} k(s-t, \sigma, \xi) x(s, \sigma) d \sigma d s\right] \\
\quad=(A(t) x(t))(\xi)+\int_{-\infty}^{t} \int_{0}^{1} e^{-c_{2}(t-s)} b(s-t) \mathcal{G}(t, x(s, \xi)) d \sigma d s \\
\quad t \in[0, T], \xi \in[0,1] \\
x(t, 0)=x(t, 1)=0, \quad t \in[0, T] \\
\Delta x\left(t_{i}, \cdot\right)=x\left(t_{i}^{+}, \cdot\right)-x\left(t_{i}^{-}, \cdot\right)=\int_{0}^{1} p_{i}\left(x\left(t_{i}, \sigma\right), \cdot\right) d \sigma, \\
x(s, \xi)=\varphi(s, \xi) \in \mathcal{B}, \quad s \leq 0,0 \leq \xi \leq 1,
\end{array}\right.
$$

where $c_{1}$ and $c_{2}$ are positive constants with $0<\gamma<\min \left\{c_{1}, c_{2}\right\}$. Define the functions $F, G$ : $[0, T] \times \mathcal{B} \rightarrow X$ and $I_{i}: \mathcal{B} \rightarrow X$ by

$$
\begin{aligned}
& F(t, \phi)(\xi)=\int_{-\infty}^{0} \int_{0}^{1} e^{c_{1} s} k(s, \sigma, \xi) \phi(s, \sigma) d \sigma d s \\
& G(t, \phi)(\xi)=\int_{-\infty}^{0} e^{c_{2} s} b(s) \mathcal{G}(t, \phi(s, \xi)) d s, \\
& I_{i}(\phi)(\xi)=\int_{0}^{1} p_{i}(\phi(0, \sigma), \xi) d \sigma .
\end{aligned}
$$

It is clear that problem (4.3) can be modeled as the abstract impulsive Cauchy problem (1.1). To treat this system, we assume the following conditions:

(C1) $k:(-\infty, 0] \times[0,1] \times[0,1] \rightarrow \mathbb{R}$ is square integrable with $k(s, \sigma, 0)=k(s, \sigma, 1)=0$ for all $s \in(-\infty, 0]$ and $\sigma \in[0,1]$. Moreover, $k(s, \sigma, \xi)$ is twice differentiable with respect to $\xi$ and

$$
c_{0}:=\sum_{i=0}^{2}\left(\int_{0}^{1} \int_{-\infty}^{0} \int_{0}^{1}\left|\frac{\partial^{i}}{\partial \xi^{i}} k_{1}(s, \sigma, \xi)\right|^{2} d \sigma d s d \xi\right)^{\frac{1}{2}}<\infty .
$$


(C2) The function $b:(-\infty, 0] \rightarrow \mathbb{R}$ is measurable such that the function $s \mapsto e^{\left(c_{2}-\gamma\right) s} b(s)$ is integrable on $(-\infty, 0]$ and we put

$$
\kappa=\int_{-\infty}^{0} e^{\left(c_{2}-\gamma\right) s}|b(s)| d s
$$

(C3) $\mathcal{G}:[0, T] \times \mathcal{B} \rightarrow 2^{X}$ is a multimap with compact and convex values and there exist a function $\mu \in L^{1}([0, T])$ and a function $\vartheta \in C_{b}((-\infty, 0], \mathbb{R})$ such that

$$
\|\mathcal{G}(t, \phi(s, \xi))\| \leq \mu(t)|\vartheta(s)|\|\phi(s)\|, \quad 0 \leq t \leq T, s \leq 0,0 \leq \xi \leq 1 .
$$

Moreover, we suppose that $\mathcal{G}$ is compact in its second argument.

(C4) The functions $p_{i}: \mathbb{R} \times[0,1] \rightarrow \mathbb{R}, i=1,2, \ldots, m$, are continuous, and there are positive constants $l_{i}$ such that

$$
\left|p_{i}\left(\tau_{1}, \xi\right)-p_{i}\left(\tau_{2}, \xi\right)\right| \leq l_{i}\left|\tau_{1}-\tau_{2}\right|, \quad \xi \in[0,1], \tau_{1}, \tau_{2} \in \mathbb{R}
$$

The next result is a consequence of Theorem 3.1.

Theorem 4.1 Suppose that the previous conditions (C1)-(C5) hold. If

$$
\left[\left(1+M_{0} \tilde{A}\right) c_{0}+M_{0}\left(\kappa\|\vartheta\|_{\infty}\|\mu\|_{L^{1}([0, T])}+\sum_{i=1}^{m} l_{i}\right)\right]<1,
$$

then there is a mild solution of the impulsive system (4.3).

Proof Observe that

$$
\|A(s) U(t, s) \psi\|=\|U(t, s) A(s) \psi\| \leq\|U(t, s)\|_{L(X)}\|A(s)\|_{L(D, X)}\|\psi\|_{D}, \quad \psi \in D,
$$

and hence

$$
\|A(s) U(t, s)\|_{L(D, X)} \leq\|U(t, s)\|_{L(X)}\|A(s)\|_{L(D, X)} \leq M \sup _{s \in[0, T]}\|A(s)\|_{L(D, X)} .
$$

By (C1), it is easy to see that $F(t, \phi) \in D$ for all $(t, \phi) \in[0, T] \times \mathcal{B}$, and assumption (H1) is thus fulfilled by taking

$$
\mathcal{H}(t-s)=\sup _{0 \leq s<t \leq T}\|U(t, s)\|_{L(X)} \cdot \sup _{s \in[0, T]}\|A(s)\|_{L(D, X)}=M_{0} \tilde{A} .
$$

The following estimate, which follows from $(\mathrm{C} 1)$, verifies that $F$ satisfies $(\mathrm{H} 2)$ :

$$
\begin{aligned}
\|F(t, \phi)\|^{2} & =\int_{0}^{1}\left[\int_{-\infty}^{0} \int_{0}^{1} e^{c_{1} s} k_{1}(s, \sigma, \xi) \phi(s, \sigma) d \sigma d s\right]^{2} d \xi \\
& =\int_{0}^{1}\left[\int_{-\infty}^{0} e^{c_{1} s}\left(\int_{0}^{1} k_{1}(s, \sigma, \xi) \phi(s, \sigma) d \sigma\right) d s\right]^{2} d \xi \\
& \leq \int_{0}^{1}\left[\int_{-\infty}^{0} e^{c_{1} s}\left(\int_{0}^{1}\left|k_{1}(s, \sigma, \xi)\right|^{2} d \sigma\right)^{1 / 2}\left(\int_{0}^{1}|\phi(s, \sigma)|^{2} d \sigma\right)^{1 / 2} d s\right]^{2} d \xi
\end{aligned}
$$




$$
\begin{aligned}
& \leq \int_{0}^{1}\left[\int_{-\infty}^{0} e^{\left(c_{1}-\gamma\right) s}\left(\int_{0}^{1}\left|k_{1}(s, \sigma, \xi)\right|^{2} d \sigma\right)^{1 / 2} e^{\gamma s}\|\phi(s)\| d s\right]^{2} d \xi \\
& \leq\left(c_{0}\|\phi\|_{\mathcal{B}}\right)^{2} .
\end{aligned}
$$

Moreover, take $g \in G(\cdot, \phi)$, and we have

$$
\begin{aligned}
\|g(t)\|^{2} & =\int_{0}^{1}\left(\int_{-\infty}^{0} e^{c_{2} s} b(s) g(t, \phi(s, \xi)) d s\right)^{2} d \xi \\
& =\int_{0}^{1}\left(\int_{-\infty}^{0} e^{c_{2} s} b(s) \mu(t)|\vartheta(s)|\|\phi(s)\| d s\right)^{2} d \xi \\
& \leq\left(\mu(t)\|\vartheta\|_{\infty} \int_{-\infty}^{0} e^{\left(c_{1}-\gamma\right) s} b(s) e^{\gamma s}\|\phi(s)\| d s\right)^{2} \\
& \leq\left(\kappa\|\vartheta\|_{\infty} \mu(t)\|\phi\|_{\mathcal{B}}\right)^{2},
\end{aligned}
$$

yielding

$$
\|G(t, \phi)\| \leq \kappa\|\vartheta\|_{\infty} \mu(t)\left(1+\|\phi\|_{\mathcal{B}}\right) .
$$

This shows that $G$ satisfies (H3). From condition (C4), we have

$$
\begin{aligned}
\left|I_{1}(\phi)(\xi)-I_{i}(\psi)(\xi)\right| & \leq \int_{0}^{1}\left|p_{i}(\phi(0, \sigma), \xi)-p_{i}(\psi(0, \sigma), \xi)\right| d \sigma \\
& \leq l_{i} \int_{0}^{1}|\phi(0, \sigma)-\psi(0, \sigma)| d \sigma \\
& \leq l_{1}\|\phi(0)-\psi(0)\| \\
& \leq l_{1}\|\phi-\psi\|_{\mathcal{B}}
\end{aligned}
$$

by axiom (A)(ii) and (4.2).

Consequently, from (4.4) and Theorem 3.1, the problem (4.3) has a mild solution.

The example we provide here is nontrivial. Actually, consider the nonlinear term $\mathcal{G}$ : $[0, T] \times \mathcal{B} \rightarrow 2^{X}$ given by

$$
\mathcal{G}(t, \phi):=(2+\cos t) \Omega_{\phi}
$$

where $\Omega_{\phi}$ is the convex hull of the set

$$
\left\{\eta_{0}+\eta \mid \eta_{0} \neq 0 \in X, \eta_{0} \perp \eta,\left\|\eta_{0}\right\| \leq 1, \eta=a \phi(0), a \leq 1\right\} .
$$

It is clear that $\mathcal{G}$ has convex and compact values, $\mathcal{G}$ is locally compact, and $G$ is compact in its second argument. Moreover, $\mathcal{G}(t, \varphi) \neq 0$ even if $\varphi \equiv 0$.

Now, let $\left\{\phi_{n}\right\}$ be a sequence in $\mathcal{B}=C_{\gamma}$ such that $\phi_{n} \rightarrow \phi$ in $\mathcal{B}=C_{\gamma}$ and let $\eta_{n}=a \phi_{n}(0)$ for all $n \in \mathbb{N}$. Since

$$
\left\|\eta_{n}-\eta\right\| \leq a\left\|\phi_{n}(0)-\phi(0)\right\| \leq a\left\|\left(\phi_{n}\right)_{0}-\phi_{0}\right\|_{\mathcal{B}} \rightarrow 0 \quad \text { as } n \rightarrow \infty,
$$


$\mathcal{G}$ is closed, and hence $\mathcal{G}$ is upper semi-continuous (see [22], p.5). Therefore, $\mathcal{G}$ satisfies the conditions (H3) and (H4). Thus, if inequality (4.4) holds with suitable functions $\vartheta, \mu$, $k, b$, and $p_{i}$ satisfying (C1), (C2), and (C4), then the system (4.3) considered in Section 4 has a nontrivial solution in this case, since for $x=0, t \in[0, T]$, and $\xi \in[0,1], \frac{\partial}{\partial t} x(t, \xi)=$ $(A(t) x(t))(\xi)=0$, and we have

$$
\int_{-\infty}^{t} \int_{0}^{1} e^{-c_{1}(t-s)} k(s-t, \sigma, \xi) x(s, \sigma) d \sigma d s=0
$$

and

$$
\int_{-\infty}^{t} \int_{0}^{1} e^{-c_{2}(t-s)} b(s-t) g(t, x(s, \xi)) d \sigma d s \neq 0
$$

for any selection $g \in \mathcal{G}$ and suitably selected $b$.

\section{Competing interests}

The authors declare that they have no competing interests.

\section{Authors' contributions}

All authors contributed equally and significantly in writing this article. All authors read and approved the final manuscript.

\section{Author details}

'School of Computer and Information Engineering, Xiamen University of Technology, Xiamen, 361024, China. ${ }^{2}$ Department of Information Management, Yuan Ze University, Chung-Li, 32003, Taiwan. ${ }^{3}$ Department of Information Management, Innovation Center for Big Data and Digital Convergence, Yuan Ze University, Chung-Li, 32003, Taiwan.

\section{Acknowledgements}

In this research, the first author was partially supported by Xiamen University of Technology's International Cooperation and Exchange Project under Grant No. E201300200, and Fujian Province Department of Education Category A projects under Grant No. JA14243. The second author and third author were supported partly by the National Science Council of the Republic of China.

Received: 14 August 2015 Accepted: 13 December 2015 Published online: 30 December 2015

\section{References}

1. Hale, J, Kato, J: Phase space for retarded equations with infinite delay. Funkc. Ekvacioj 21(1), 11-41 (1978)

2. Bainov, DD, Simeonov, PS: Systems with Impulsive Effect. Horwood, Chichester (1989)

3. Benchohra, M, Henderson, J, Ntouyas, SK: Impulsive Differential Equations and Inclusions. Hindawi Publishing Corporation, New York (2006)

4. Haddad, WM, Chellabonia, V, Nersesov, SG, Sergey, G: Impulsive and Hybrid Dynamical Systems: Stability, Dissipativity and Control. Princeton University Press, Princeton (2006)

5. Lakshmikantham, V, Bainov, DD, Simeonov, PS: Theory of Impulsive Differential Equations. World Scientific, Singapore (1989)

6. Samoilenko, AM, Perestyuk, NA, Simeonov, PS: Impulsive Differential Equations. World Scientific, Singapore (1995)

7. Benchohra, M, Henderson, J, Ntouyas, SK: Existence results for impulsive multivalued semilinear neutral functional inclusions in Banach spaces. J. Math. Anal. Appl. 263, 763-780 (2001)

8. Hernández, E, Henríquez, HR: Impulsive partial neutral differential equations. Appl. Math. Lett. 19, 215-222 (2006)

9. Rogovchenko, YV: Nonlinear impulse evolution systems and applications to population models. J. Math. Anal. Appl. 207, 300-315 (1997)

10. Chukwu, EN: Differential Models and Neutral Systems for Controlling the Wealth of Nations. World Scientific, River Edge (2001)

11. Lunardi, A, Song, W: On the linear heat equation with fading memory. SIAM J. Math. Anal. 21, 1213-1224 (1990)

12. Cannarsa, P, Sforza, D: Global solutions of abstract semilinear parabolic equations with memory terms. Nonlinear Differ. Equ. Appl. 10, 399-430 (2003)

13. Gurtin, ME, Pipkin, AC: A general theory of heat conduction with finite wave speeds. Arch. Ration. Mech. Anal. 31, 113-126 (1968)

14. Nunziato, J: On heat conduction in materials with memory. Q. Appl. Math. 29, 187-204 (1971)

15. McCamy, R: An integrodifferential equation with applications in heat flow. Q. Appl. Math. 35, 1-19 (1977)

16. Hernández, E, Henríquez, H: Existence of periodic solutions of partial neutral functional differential equations with unbounded delay. J. Math. Anal. Appl. 221(2), 499-522 (1998)

17. Hernández, E, Henríquez, H: Existence results for partial neutral functional differential equations with unbounded delay. J. Math. Anal. Appl. 221(2), $452-475$ (1998)

18. Diagana, T, Hernández, E, Rabelo, M: Pseudo almost periodic solutions to some non-autonomous neutral functional differential equations with unbounded delay. Math. Comput. Model. 45, 1241-1252 (2007) 
19. Cuevas, C, Hernández, E, Rabelo, M: The existence of solutions for impulsive neutral functional differential equations. Comput. Math. Appl. 58, 744-757 (2009)

20. Li, W-S, Chang, Y-K, Nieto, JJ: Solvability of impulsive neutral evolution differential inclusions with state-dependent delay. Math. Comput. Model. 49, 1920-1927 (2009)

21. Ke, TD, Obukhovskii, V, Wong, N-C, Yao, J-C: Approximate controllability for systems governed by nonlinear Volterra type equations. Differ. Equ. Dyn. Syst. 20, 35-52 (2012)

22. Kamenskii, M, Obukhovskii, V, Zecca, P: Condensing Multivalued Maps and Semilinear Differential Inclusions in Banach Spaces. de Gruyter, Berlin (2001)

23. Papageorgious, N, Huang, N-J: On the theory of Banach space valued multifunctions. I. Integration and conditional expectation. J. Multivar. Anal. 17, 185-206 (1985)

24. Pazy, A: Semigroups of Linear Operators and Applications to Partial Differential Equations. Springer, New York (1983)

25. Andres, J, Górniewicz, L: Topological Fixed Point Principles for Boundary Value Problems. Kluwer Academic, Dordrecht (2003)

26. Klein, E, Thomson, A: Theory of Correspondences. Wiley, New York (1984)

27. Papageorgiou, NS: Convergence theorems for Banach space valued integrable multifunctions. Int. J. Math. Math. Sci. 10, 433-442 (1987)

28. Lunardi, A: Analytic Semigroups and Optimal Regularity in Parabolic Problems. Birkhäuser, Basel (1995)

29. Hino, Y, Murakami, S, Naito, T: Functional Differential Equations with Infinite Delay. Lecture Notes in Math., vol. 1473. Springer, Berlin (1991)

\section{Submit your manuscript to a SpringerOpen ${ }^{\circ}$ journal and benefit from:}

- Convenient online submission

- Rigorous peer review

Immediate publication on acceptance

Open access: articles freely available online

- High visibility within the field

- Retaining the copyright to your article 